\title{
Ferropenia: no siempre son pérdidas
}

\author{
C. ALONSO COTONER, F. CASELLAS JORDÁ, Ma L. CHICHARRO SERRANO, \\ I. DE TORRES RAMÍREZ', J. R. MALAGELADA BENAPRÉS
}

Servei de Digestiu. 'Departament d'Anatomia Patològica. Hospital Universitari Vall d'Hebron. Barcelona

\author{
IRON DEFICIENCY: NOT ALWAYS BLOOD LOSSES
}

\section{RESUMEN}

La ferropenia puede ser la única manifestación de un síndrome malabsortivo, secundario a la presencia de una atrofia intestinal. Por ello, descartadas las pérdidas gastrointestinales, debería tenerse en cuenta esta posibilidad en el estudio de la ferropenia.

Objetivos: Evaluar la utilidad de la biopsia yeyunal en el diagnóstico de la malabsorción intestinal como causa de ferropenia y analizar cuales son las causas de ferropenia malabsortiva.

Método: Se han incluido 70 pacientes con ferropenia (ferritina $<25$ ng/ml) remitidos a la Unitat de Proves Funcionals Digestives, para la valoración de una posible malabsorción intestinal mediante la realización de una biopsia yeyunal. La biopsia yeyunal se obtuvo distal al ángulo de Treitz mediante cápsula de Watson. Se clasificaron los resultados histológicos según los criterios de Marsh.

Resultados: La biopsia yeyunal se realizó en 66 pacientes. Fue normal en 25 pacientes, inespecífica en 1 , en 12 se evidenciaron lesiones grado 1 (infiltrado linfocitario), en 2 lesiones grado 2 (hiperplasia de criptas) y en 25 lesiones grado 3 (atrofia). En otro paciente se objetivó una giardiasis intestinal. El diagnóstico clínico de los pacientes incluídos fue de enfermedad celíaca en $21(32 \%)$, aclorhidria en $7(10,5 \%)$, sobrecrecimiento bacteriano en $1(1,5 \%)$, giardiasis intestinal en otro $(1,5 \%)$, pérdidas ginecológicas en otra $(1,5 \%)$ y $35(53 \%)$ quedaron sin diagnóstico definitivo.

Conclusiones: La biopsia yeyunal es una prueba útil en el diagnóstico de malabsorción como causa de ferropenia, permitiendo poner de manifiesto la existencia de patología intestinal en un alto porcentaje de casos.

PALABRAS CLAVE: Ferropenia. Anemia. Biopsia yeyunal. Malabsorción.
ABSTRACT

Iron deficiency may be the only presenting sign of malabsorption due to the presence of intestinal villous atrophy. Once gastrointestinal blood losses have been excluded, intestinal malabsorption should be investigated.

Objectives: To determine the utility of jejunal biopsy in the evaluation of iron deficiency due to a defective absorption and the different diseases that may cause malabsortive iron deficiency.

Methods: Seventy patients with iron deficiency (ferritin $<25 \mathrm{ng} / \mathrm{ml}$ ), referred to the Unitat de Proves Funcionals Digestives to perform a jejunal biopsy were included. Jejunal biopsy was obtained distal to Treitz with a Watson capsule. Histological changes were classified according to the criteria proposed by Marsh.

Results: Jejunal biopsy was performed in 66 patients. Histology was normal in 25, unespecific in 1, showed inflammatory infiltrate in 12, hyperplastic changes in 2 and atrophy in 25 . In one patient showed intestinal giardiasis. The clinical diagnosis was celiac sprue in 21 patients (32\%), aclorhydria in $7(10.5 \%)$, bacterial overgrowth in $1(1.5 \%)$, intestinal giardiasis in 1 (1.5\%), menstrual blood loss in 1 1.5\%) and 35 (53\%) patients remained without a definitive diagnosis.

Conclusions: Jejunal biopsy is useful in the evaluation of iron deficiency due to intestinal malabsorption and reveals intestinal abnormalities in a significant number of cases.

KEY WORDS: Iron deficiency. Anemia. Jejunal biopsy. Malabsorption.

Alonso Cotoner C, Casellas Jordá F, Chicharro Serrano $M^{a} L$, de Torres Ramírez I, Malagelada Benaprés JR. Ferropenia: no siempre son pérdidas. An Med Interna (Madrid) 2003; 20: 227-231.

\section{INTRODUCCIÓN}

La ferropenia es uno de los déficits nutricionales más frecuentes, no sólo en los países subdesarrollados, sino también en los países ricos. Según la OMS, el $15 \%$ de la población mundial podría tener ferropenia, lo que supone unos 500-600 millones de personas en el mundo (1). Se estima que hasta un
5-10\% de las mujeres premenopáusicas presenta anemia ferropénica (2), siendo su causa más frecuente el aumento de las pérdidas menstruales, aunque también son causas habituales el embarazo y la lactancia (3). En el caso de los hombres adultos y mujeres postmenopáusicas del mundo desarrollado, la prevalencia de la anemia ferropénica es de un $2-5 \%$ (4). En las mujeres postmenopáusicas y los varones, la causa más fre-

Trabajo aceptado: 24 de enero de 2003

Correspondencia: Francesc Casellas. Servei de Digestiu. Hospital Universitari Vall d'Hebron. Pg. Vall d'Hebron, 119. 08035 Barcelona 
cuente de anemia ferropénica es la pérdida crónica de pequeñas cantidades de sangre, la mayoría de veces de origen digestivo (5-7). Por ello, la anemia ferropénica constituye un motivo frecuente de consulta en gastroenterología, que se ha cifrado en un 4 a un $13 \%$ según las series (8). Sin embargo, una vez descartadas las pérdidas gastrointestinales, debe tenerse en cuenta que la ferropenia puede tener otras causas digestivas. En este sentido, no hay que olvidar que la anemia ferropénica puede ser la única manifestación de un síndrome de malabsorción intestinal $(5,8,9)$. Por este motivo siempre debe sospecharse la posibilidad de una malabsorción intestinal ante una anemia ferropénica de causa no aparente. Sin embargo, en la clínica asistencial en ocasiones no se realizan las pruebas adecuadas de estudio de la absorción intestinal, lo que puede derivar en retrasos diagnósticos o en pacientes no diagnosticados. En muchos casos de malabsorción intestinal, la sospecha se confirma mediante la realización de pruebas de cribado (como el test de xilosa, etc.) y se llega al diagnóstico etiológico a través del análisis histológico de biopsias de duodeno distal o yeyuno (10). El objetivo de nuestro estudio ha sido evaluar la utilidad de la biopsia yeyunal en el diagnóstico de la malabsorción intestinal como causa de ferropenia y, al mismo tiempo, analizar cuales son las causas más frecuentes de ferropenia malabsortiva.

\section{MATERIAL Y MÉTODOS}

\section{PACIENTES}

Se han incluido los pacientes remitidos a la Unitat de Proves Funcionals Digestives en el periodo 1991-2001 para el estudio de ferropenia de origen no atribuible a pérdidas hemáticas. Se incluyeron los pacientes con un nivel de ferritina sérica inferior a $25 \mathrm{ng} / \mathrm{ml}$, independientemente de que se acompañara de anemia ferropénica. Se excluyeron del estudio los pacientes en los que se objetivaron pérdidas digestivas mediante la realización de tests repetidos de sangre oculta en heces. Para el estudio se recogieron los datos clínicos (edad, sexo, menopausia, astenia, palidez, presencia de diarrea, pérdida de peso, respuesta a hierro oral, consumo habitual de antiinflamatorios no esteroideos, antecedentes de gastrectomía o resección intestinal) y analíticos (hemoglobina, hematocrito, volumen corpuscular medio, ferritina, vitamina B12, ácido fólico, transferrina, tiempo se protrombina, calcio, albúmina, colesterol), así como el resto de exploraciones realizadas durante el estudio de la ferropenia (fibrogastroscopia, fibrocolonoscopia, anticuerpos anticélula parietal, quimismo gástrico, test del aliento de hidrógeno con glucosa, test del aliento de hidrógeno con xilosa, test de Schilling) y biopsia yeyunal.

\section{MÉTODO}

La biopsia yeyunal se obtuvo distal al ángulo de Treitz mediante cápsula de Watson colocada bajo control radioscópico. La muestra obtenida se procesó según técnica de rutina, mediante fijación en formol y tinción con hematoxilina-eosina. El análisis histológico de las muestras fue llevado a cabo por un patólogo experto que desconocía los datos clínicos del paciente. Los resultados se clasificaron atendiendo a la clasifi- cación propuesta por Marsh (11), según la existencia de linfocitosis intraepitelial (lesión tipo 1), linfocitosis e hiperplasia de las criptas (tipo 2), atrofia vellositaria (tipo 3), atrofia vellositaria e hipoplasia de las criptas (tipo 4) o según la existencia de hallazgos específicos.

La ferropenia se atribuyó a la existencia de enfermedad celíaca cuando tras una biopsia patológica compatible, se objetivó mejoría histológica y/o clínico-analítica tras seguir una dieta sin gluten. La ferropenia se atribuyó a pérdidas ginecológicas en aquellas pacientes en las que la ferropenia remitió tras la menopausia o tras la histerectomía por patología uterina, siendo además el resto de exploraciones (fibrogastroscopia, fibrocolonoscopia y biopsia yeyunal) negativas. La aclorhidria se consideró como la causa de la ferropenia en aquellos pacientes que presentaron un quimismo gástrico compatible, antecedentes de gastrectomía o presencia de atrofia gástrica en la biopsia con niveles de gastrina elevados, siendo además el resto de exploraciones normales.

\section{ESTADÍSTICA}

Los datos referidos a variables cuantitativas contínuas se expresan como media \pm error estándar, tras comprobarse que seguían una distribución normal (test de Kolmogorov-Smirnoff). Las variables cualitativas se expresan como porcentaje. Para determinar la existencia de diferencias estadísticas entre medias, se utilizó el test de la t de Student.

\section{RESULTADOS}

\section{PACIENTES}

Inicialmente entraron en el estudio 70 pacientes. Cuatro pacientes fueron retirados del estudio por presentar pérdidas hemáticas durante el estudio, detectadas por positivización del test de sangre oculta en heces, con lo que se incluyeron en total 66 pacientes.

Los principales datos epidemiológicos se resumen en la tabla I. En la misma tabla se detallan los síntomas que motivaron el estudio. Cabe destacar que los síntomas se relacionan con la presencia de ferropenia, siendo los síntomas digestivos como la diarrea poco frecuentes, ya que sólo la referían 22 de

\section{TABLA I}

CARACTERÍSTICAS DE LOS PACIENTES INCLUIDOS EN EL ESTUDIO

\begin{tabular}{lc}
\hline Edad (años) & $41 \pm 2$ \\
Hombres/mujeres & $18 / 48$ \\
M ujeres premenopáusicas & 32 \\
M ujeres menopáusicas & 12 \\
Síntomas: & \\
$\quad$ Astenia $(n=29)$ & 20 \\
Palidez $(n=28)$ & 17 \\
$\quad$ Diarrea $(n=46)$ & 22 \\
Pérdida de peso ( $n=40)$ & 16 \\
Respuesta a hierro oral $(n=34)$ & 10 sí / 24 no \\
Consumo habitual de AINEs $(n=25)$ & 8 \\
Antecedente de gastrectomía $(n=65)$ & 1 \\
\hline
\end{tabular}


los 46 pacientes en los que se registró este dato. Sin embargo, no se encontraron diferencias significativas respecto al grado de ferropenia entre los pacientes que referían síntomas frente a los que no referían sintomatología ( $\mathrm{p}>0,005)$.

En la tabla II se recogen las exploraciones practicadas a los pacientes incluídos en el estudio, así como el resultado de las mismas. En la endoscopia practicada previa a la inclusión en el estudio se objetivó la existencia de hernia hiatal en 9 pacientes, divertículos colónicos en 3 y hemorroides en 9 pacientes, pero se descartaron como causa de la ferropenia por la negatividad repetida de las pruebas de sangre en heces. Cuatro pacientes referían antecedentes de úlcera péptica, pero la endoscopia demostró cicatrización de las lesiones en los cuatro pacientes. En 38 pacientes se determinaron los anticuerpos anti-célula parietal, que fueron positivos en 7 pacientes ( 1 a título de 1/80, 2 a 1/160 y 4 a 1/320). En 3 de estos pacientes se realizó un quimismo gástrico, que demostró una aclorhidria histamino-resistente en todos ellos. En 1 paciente se realizó un test de Schilling, que fue patológico y se corrigió con factor intrínseco. En 3 pacientes se realizó biopsia gástrica, que fue demostrativa de atrofia gástrica. En un paciente se realizó una prueba del aliento de hidrógeno con glucosa que fue patológica, sugestiva de la existencia de un sobrecrecimiento bacteriano. En 63 pacientes se realizó un test del aliento con xilosa que fue patológico (sugestivo de una inadecuada integridad intestinal) en 49. En la tabla III se detallan los resultados de los análisis de laboratorio. Cabe destacar que no existían datos analíticos sugestivos de malnutrición en nuestra muestra.

TABLA II

\section{EXPLORACIO NES REALIZADAS}

\begin{tabular}{lc}
\hline Tránsito esófagogastroduodenal $(n=52)$ & 11 patológicos \\
Fibrogastroscopia $(n=38)$ & 38 normales \\
Fibrocolonoscopia $(n=30)$ & 30 normales \\
Anticuerpos anticélula parietal $(n=38)$ & 7 positivos \\
Quimismo gástrico $(n=3)$ & 3 patológicos \\
Test de glucosa $(n=1)$ & 1 patológico \\
Test de Schilling $(n=1)$ & 1 patológicos \\
Test de xilosa $(n=63)$ & 49 patológicos \\
\hline
\end{tabular}

TABLA III

\begin{tabular}{ll}
\multicolumn{2}{c}{ TABLA III } \\
\multicolumn{2}{c}{ DATOS ANALÍTICOS } \\
\hline Hemoglobina & $11,1 \pm 0,3 \mathrm{~g} / \mathrm{dl}$ \\
Hematocrito & $33,6 \pm 0,8 \%$ \\
VCM & $80,9 \pm 1,5 \mathrm{fL}$ \\
Ferritina & $9,2 \pm 1 \mathrm{ng} / \mathrm{ml}$ \\
Vitamina B12 & $404 \pm 28,4 \mathrm{pg} / \mathrm{ml}$ \\
Ácido fólico & $8,4 \pm 0,6 \mathrm{ng} / \mathrm{ml}$ \\
Transferrina & $343,7 \pm 11,3 \mathrm{mg} / \mathrm{dl}$ \\
Tiempo de protrombina & $96,5 \pm 1,9 \%$ \\
Calcio & $9,2 \pm 0,1 \mathrm{mg} / \mathrm{dl}$ \\
Albúmina & $4,2 \pm 0,1 \mathrm{~g} / \mathrm{dl}$ \\
Colesterol & $178,8 \pm 5,4 \mathrm{mg} / \mathrm{dl}$ \\
\hline
\end{tabular}

\section{BIOPSIA YEYUNAL}

La biopsia fue informada como patológica en 40 casos (60.6\%). En 12 de ellos se evidenciaron lesiones de grado 1, en 2 lesiones de grado 2 y en 25 lesiones de grado 3. En otra paciente se objetivó una giardiasis intestinal. La biopsia fue informada como normal en 25 pacientes $(37.9 \%)$ y compatible con cambios inespecíficos en 1 paciente $(1.5 \%)$.

\section{DIAGNÓSTICO ETIOLÓGICO DE LA FERROPENIA}

De los 66 pacientes incluidos en el estudio, 21 pacientes cumplieron criterios diagnósticos de enfermedad celíaca, 7 de aclorhidria, 1 de giardiasis intestinal y 1 de sobrecrecimiento bacteriano, lo que supone que un $45,5 \%$ de los pacientes presentaba una ferropenia secundaria a malabsorción, bien por defecto en la secrección de ácido clorhídrico a nivel gástrico o bien por una alteración en la integridad de la mucosa intestinal. En 35 pacientes no se llegó a ningún diagnóstico definitivo o fueron perdidos en el seguimiento.

En las 32 pacientes premenopáusicas la causa de la ferropenia fue aclorhidria en 5, celiaquía en 7 , giardiasis en una y en otra la causa fue ginecológica (hipermenorrea, con resolución de la ferropenia tras la menopausia ). De las 12 mujeres menopáusicas, 2 fueron diagnosticadas de aclorhidria, 2 de celiaquía y 1 de sobrecrecimiento bacteriano. En 4 pacientes no se pudo registrar la presencia o no de menstruación en el momento de la realización del estudio.

\section{DISCUSIÓN}

La ferropenia es el déficit nutricional más frecuente, tanto en los países desarrollados como en los subdesarrollados. En estos últimos, la causa más frecuente es el déficit de ingesta. Sin embargo, en los países desarrollados, las causas son muy distintas y difieren en función del segmento de la población estudiado. Así, en las mujeres postmenopaúsicas y mayores de 50 años en general, la causa más frecuente son las pérdidas digestivas, mientras que en las mujeres premenopáusicas lo es el aumento de las pérdidas menstruales. Por ello, en los pacientes en los que se objetiven pérdidas digestivas, bien sean macro o microscópicas, se recomienda realizar un estudio endoscópico (fibrogastroscopia y fibrocolonoscopia), lo que permite identificar la causa de la ferropenia en un 53-62\% de los casos $(6,7)$. Sin embargo, en muchas ocasiones la ferropenia no es secundaria a pérdidas crónicas, sino a malabsorción de hierro a nivel intestinal. Esto explica la importancia de evaluar la integridad funcional de la mucosa intestinal mediante las pruebas adecuadas.

En el presente estudio, de los 66 pacientes incluidos, 21 fueron diagnosticados de celiaquía, lo que supone un $32 \%$ del total. Esta elevada prevalencia se explicaría porque se trata de una muestra de pacientes seleccionados en los que previamente se habían descartado pérdidas hemáticas gastrointestinales como causa de ferropenia. Desde la descripción de la enfermedad celiaca en 1888 por Samuel Gee (12), se han descrito diferentes formas de presentación de la enfermedad. Aunque en las series clínicas clásicas la existencia de diarrea y síntomas malabsortivos es frecuente $(13,14)$, cada vez es más habitual que la enfermedad se diagnostique en una fase clínica- 
mente silente. De hecho, aproximadamente el 50\% de los adultos no tiene diarrea, siendo la anemia ferropénica una de las formas de presentación más habituales (15). El examen histológico de la mucosa intestinal sigue siendo la base del diagnóstico de la enfermedad. En 1992, Marsh describió una clasificación de los hallazgos anatomopatológicos en función de la severidad de la lesión histológica yeyunal (11). Atendiendo a esta clasificación existen 4 grados de lesión. El tipo 1 se caracteriza por un aumento de los linfocitos intraepiteliales, lo que ocurre en un $40 \%$ de los pacientes con dermatitis herpetiforme y en un $10 \%$ de los familiares de primer grado de pacientes celíacos (11). Esta lesión no se asocia con la presencia de malabsorción y los estudios de permeabilidad intestinal en estos pacientes pueden ser normales (16). Doce de los pacientes incluidos en el estudio presentaron esta lesión en la biopsia, pero sólo 2 fueron diagnosticados de enfermedad celíaca. En la lesión tipo 2, además del incremento de linfocitos intraepiteliales, existe una hiperplasia de las criptas, pero sin disminución de la altura de las vellosidades, lo que se aprecia en un $20 \%$ de los pacientes con dermatitis herpetiforme o enfermedad celíaca no tratados. Dos pacientes presentaron esta lesión en la biopsia. En ninguno de ellos se llegó a un diagnóstico definitivo, por pérdida en el seguimiento. La lesión tipo 3 corresponde a la clásica lesión de la enfermedad celíaca. Consiste en una atrofia de las vellosidades, añadida a los cambios descritos en las lesiones 1 y 2. Sin embargo, como se ha apuntado antes, aunque la lesión es característica, no es exclusiva, pudiendo aparecer en enfermedades tan diversas como la giardiasis intestinal severa, la enfermedad injerto contra huésped, isquemia crónica del intestino delgado o esprue tropical (16). En 25 pacientes se encontraron lesiones tipo 3, de los cuales 18 fueron diagnosticados de celiaquía. A pesar de la severidad de la lesión, muchos de los pacientes permenecen asintomáticos, como ocurrió en nuestro estudio, en el que muy pocos pacientes presentaban una clínica sugestiva de malabsorción. Por último, la lesión tipo 4 corresponde al estadío final de la enfermedad en aquellos pacientes que no responden a la dieta sin gluten. Se caracteriza por la presencia de atrofia vellositaria e hipoplasia de las criptas. Ninguno de los pacientes incluídos en el estudio presentó lesiones compatibles con este estadío.

De los 27 pacientes restantes, un paciente fue diagnosticado de giardiasis masiva y en el resto las biopsias fueron normales o inespecíficas. Aunque la atrofia vellositaria y la giardiasis pueden justificar la existencia de ferropenia, en los casos con lesiones tipo 1 y 2 de Marsh es más difícil establecer si los hallazgos histológicos son la causa de la ferropenia. En nuestro estudio se realizó un test del aliento con xilosa que fue patológico en los 14 pacientes con lesiones tipo 1 y 2 , lo que podría significar que no siempre existe una adecuada integridad de la mucosa intestinal en estos pacientes. En estos dos grupos habría sido de gran utilidad la realización de una segunda biopsia tras dieta sin gluten, con el objeto de determinar la presencia o no de una mejoría histológica y poder llegar a un diagnóstico definitivo. La mayoría de estos pacientes quedó sin diagnóstico, bien porque se perdieron en el seguimiento o porque a pesar del estudio no se encontró ninguna causa que justificase la ferropenia. También habría sido de utilidad la determinación de los anticuerpos antigliadina y antiendomisio, actualmente la primera prueba de cribado ante la sospecha de enfermedad celíaca. Sin embargo, en el momento del estudio no se encontraban disponibles en nues- tro centro. En cualquier caso, aún a pesar de que hubiesen sido positivos, dichos anticuerpos, en particular los anticuerpos antigliadina (AGA), pueden estar aumentados en otras enfermedades como la intolerancia a la leche de vaca, la nefropatía IgA, la enfermedad de Crohn, la enteritis eosinofílica, el esprue tropical o incluso en personas normales y ser negativos en un 2-3\% de los pacientes celíacos con déficit de $\operatorname{IgA}$, al igual que ocurriría con los anticuerpos antiendomisio (EMA) (16). Por ello, la realización de los anticuerpos es útil en el cribado de enfermedad celíaca, pero en ningún caso sustituyen a la biopsia intestinal. De la misma manera, también es importante recalcar que aunque la mayoría de los pacientes con ferropenia secundaria a malabsorción intestinal son celíacos, existen otras muchas causas que aunque poco frecuentes, sólo pueden diagnosticarse mediante la biopsia intestinal.

La realización de la biopsia yeyunal en el estudio de la ferropenia no se recomendaba de forma rutinaria (17). Sin embargo, en los últimos años se ha establecido claramente su indicación (2). Hasta 1982 en que se describió la utilidad de la biopsia endoscópica (18), el estudio histológico de la mucosa intestinal se llevaba a cabo mediante biopsias yeyunales. Son muchos los estudios que desde entonces han comparado ambas técnicas (19-22). La mayoría de ellos no ha encontrado diferencias significativas en el rendimiento de ambas, siempre y cuando la biopsia endoscópica se realice siguiendo unas determinadas recomendaciones como tomar al menos tres muestras, de duodeno distal, y con un fórceps mayor de 2 milímetros (22). Puesto que la realización de una fibrogastroscopia forma parte del estudio inicial de una anemia ferropénica, en el mismo acto diagnóstico debería llevarse a cabo la biopsia intestinal, como se aconseja en las últimas recomendaciones de la British Society of Gastroenterology (2). Sin embargo, las biopsias con cápsula siguen siendo la forma de obtener las muestras más representativas de mucosa intestinal (16), por el mayor tamaño de la muestra y la posibilidad de orientarla sobre papel.

A pesar de que la gran mayoría de las causas de malabsorción de hierro residen en la alteración de la mucosa intestinal, puede existir una alteración de la absorción de hierro de causa no intestinal. Es el caso de la gastritis crónica atrófica. Considerada hasta ahora como una causa rara de ferropenia (23), cada vez es más frecuente el diagnóstico de esta enfermedad en el estudio etiológico de la ferropenia. De hecho, hasta un $20 \%$ de los pacientes con anemia ferropénica tienen gastritis atrófica como única causa (24) e incluso se habla de que hasta en el $50 \%$ de los pacientes con gastritis crónica atrófica la anemia ferropénica es la única manifestación clínica (25). En estos pacientes, la transformación ferrosa del hierro ingerido se encuentra alterada por la ausencia de ácido clorhídrico, no siendo posible su absorción al llegar al intestino proximal. En nuestro estudio, 7 pacientes fueron diagnosticados de aclorhidria como causa de ferropenia malabsortiva, lo que supone casi un $11 \%$ del total. Uno de ellos presentaba en la biopsia intestinal hallazgos compatibles con atrofia intestinal, lo que podría ser secundario a la presencia de un sobrecrecimiento bacteriano dada la predisposición de estos pacientes a presentarlo.

En los últimos años, estudios recientes sugieren que la infección por Helicobacter pylori podría ser la responsable de una anemia ferropénica por déficit de absorción del hierro (26-28). Varios podrían ser los mecanismos responsables: la reducción en la producción de ácido clorhídrico y de ácido 
ascórbico o la presencia de proteínas fijadoras de hierro similares a la ferritina en la superficie de ciertas cepas de esta bacteria, que competirían por la fijación del hierro (28). En nuestro estudio no se pudo recoger este dato.

El sobrecrecimiento bacteriano no suele asociarse a la presencia de ferropenia. Sin embargo, en algunos pacientes puede existir un déficit de hierro secundario a la pérdida de sangre a través del tracto gastrointestinal, como resultado de la presencia de áreas ulceradas en las asas estancadas (29). Además, si bien en general no se ven alteraciones histológicas notables de la mucosa intestinal en estos pacientes, los hallazgos de la biopsia a menudo son anormales, pudiéndose observar desde infiltración de la lámina propia por linfocitos, plasmocitos o polimorfonucleares, hasta atrofia de la mucosa (29). En nuestro estudio, un paciente fue diagnosticado de sobrecrecimiento bacteriano como causa de ferropenia, objetivándose en la biopsia atrofia vellositaria. En este paciente el tratamiento antibiótico cíclico consiguió el control del sobrecrecimiento bacteriano y la recuperación de la ferropenia.
De las 48 mujeres participantes en el estudio, 32 eran premenopáusicas, 12 menopáusicas y en 4 no se pudo recoger este dato. De las 32 premenopáusicas, 13 (41\%) fueron diagnosticadas de malabsorción como causa de ferropenia ( 7 celíacas, 5 gastritis atróficas y 1 giardiasis intestinal), lo que confirma la importancia de investigar la etiología de la ferropenia en este grupo, como se ha sugerido ya en otros estudios (30) y no caer en el error de atribuirla al aumento de las pérdidas menstruales.

En conclusión, este estudio demuestra la necesidad de investigar otras causas de ferropenia, una vez excluídas las pérdidas gastrointestinales, puesto que cada vez es más frecuente el diagnóstico de una malabsorción subyacente como causa de la ferropenia. La biopsia yeyunal fue la prueba definitiva para confirmar la sospecha de malabsorción en la mayoría de éstos pacientes. Sin embargo, en los casos de aclorhidria fue necesaria la realización de otras exploraciones complementarias para llegar al diagnóstico.

\section{Bibliografía}

1. De Maeyer E, Adiec S, Tegman M. The prevalence of anaemia in the world. World Health Stat Q 1985; 38: 302-16.

2. Goddard A F, McIntyre AS, Scott BB. Guidelines for the management of iron deficiency anaemia. Gut 2000; 46 (Supl. IV): iv1-iv5.

3. Allen LH. Pregnacy and iron deficiency: unresolved issues. Nutr Rev 1997; 55: 91-101.

4. Sayer JM, Long RG. A perspective on iron deficiency anaemia. Gut 1993; 34: 1297-9.

5. Kepczyk T, Kadakia SC. Prospective evaluation of gastrointestinal tract in patients with iron-deficiency anaemia. Dig Dis Sci 1995; 40: 1283-9.

6. Rockey DC, Cello JP. Evaluation of the gastrointestinal tract in patients with iron-deficiency anaemia. N Engl J Med 1993; 329: 1691-5.

7. Zuckerman G, Benítez J. A prospective study of bidireccional endoscopy (colonoscopy and upper endoscopy) in the evaluation of patients with occult gastrointestinal bleeding. Am J Gastroenterol 1992; 87: 626.

8. McIntyre AS, Long RG. Prospective survey of investigations in outpatients referred with iron deficiency anaemia. Gut 1993; 34: 1102-7.

9. Corazza GR, Valentini RA, Andreani ML, et al. Subclinical coeliac disease is a frequent cause of iron deficiency anaemia. Scand J Gastroenterol 1995; 30: 153-6.

10. Crosby WH, Kugler HW. Intraluminal biopsy of the small intestine. Am J Dig Dis 1957; 2: 236-41.

11. Marsh MN. Gluten, major histocompatibility complex, and the small intestine. A molecular and inmunobiological approach to the spectrum of gluten sensitivity ("celiac disease"). Gastroenterol 1992; 102: 33054.

12. Gee S. On the celiac disease. St Bart Hosp Rep 1888; 24: 17-20.

13. Casellas F, Accarino A, Salas A, López Vivancos J, Guarner L. Celiac disease in the adult. Med Clin (Barc) 1985; 84: 46-50.

14. Paré P, Douville P, Caron D, Lagacé R. Adult celiac sprue: changes in the pattern of clinical recognition. J Clin Gastroenterol 1988; 10: 395400 .

15. Farrell RJ, Kelly CP. Celiac sprue. N Engl J Med 2002; 346: 180-8.

16. AGA Technical Review on Celiac Sprue. Gastroenterol 2001; 120: 1526-40.

17. Ahlquist DA. Approach to the patient with occult gastrointestinal bleeding. In Yamada T, Alpers DH, Owyang c, Powell DW, Silverstein FE. Eds. Textbook of gastroenterology. Philadelphia: JB Lippincott Co, 1995: 699-717.

18. Gillberg R, Kastrup W, Mobacken H, Stockbrugger R, Ahren C. Endoscopic duodenal biopsy compared with the Watson capsule from the

upper jejunum in patients with dermatitis herpetiformis. Scand J Gastroenterol 1982; 17: 305-8.

19. Vukavic T, Vuckovic N, Pavkov D. Routine jejunal endoscopic biopsy in children.Eur J Pediatr 1996; 155: 1002-4.

20. Oderda G, Ansaldi N. Comparison of suction capsule and endoscopic biopsy of small bowel mucosa. Gastrointest Endosc 1987; 33: 265-6.

21. Mee AS, Burke M, Vallon AG, Newman J, Cotton PB. Small bowel biopsy for malabsorption: comparison of the diagnostic adequacy of endoscopic forceps and capsule biopsy specimens. Br Med J (Clin Res Ed) 1985; 291: 769-72.

22. Branski D, Faber J, Freier S, Gottschalk-Sabag S, Shiner M. Histologic evaluation of endoscopic versus suction biopsies of small intestinal mucosae in children with and without celiac disease. J Pediatr Gastroenterol Nutr 1998; 27: 6-11.

23. Hermo JA, González L, Loureiro C. Autoimmune chronic gastritis and iron deficiency anemia. Am J Med 1999; 107: 401-3.

24. Dickey W, Kenny BD, McMillan SA, Porter KG, McConnell JB. Gastric as well as duodenal biopsies may be useful in the investigation of iron deficiency anaemia. Scand J Gastroenterol 1997; 32: 469-72.

25. Marignani M, Delle Fave G, Mecarocci S, Bordi C, Angeletti S, D'Ambra G, Aprile MR, Corleto VD, Monarca B, Annibale B. High prevalence of atrophic body gastritis in patients with unexplained microcytic and macrocytic anemia: a prospective screening study. Am J Gastroenterol 1999; 94: 766-72.

26. Annibale B, Marignani M, Monarca B, Antonelli G, Marcheggiano A, Martino G, Mandelli F, Caprilli R, Delle Fave G. Reversal of iron deficiency anemia after Helicobacter pylori eradication in patients with asymptomatic gastritis. Ann Intern Med 1999; 131: 668-72.

27. Capurso G, Marignani M, Delle Fave G, Annibale B. Iron-deficiency anemia in premenopausal women: why not consider atrophic body gastritis and Helicobacter pylori role? Am J Gastroenterol 1999; 94: 3084 5 .

28. Annibale B, Capurso G, Martino G, Grossi C, Delle Fave G. Iron deficiency anaemia and Helicobacter pylori infection. Int $\mathrm{J}$ Antimicrob Agents 2000; 16: 515-9.

29. Toskes P, Kumar A. Flora bacteriana entérica y síndrome de sobrecrecimiento bacteriano. En: Sleisenger \& Fordtran. Enfermedades gastrointestinales y hepáticas. $6^{\mathrm{a}}$ edición. Editorial Panamericana 2000; p. 16331647.

30. Bini EJ, Micale PL, Weinshel EH. Evaluation of the gastrointestinal tract in premenopausal women with iron deficiency anemia. Am J Med 1998; 105: 281-6. 